\title{
Effects of estrogen on gene expression profiles in mouse hypothalamus and white adipose tissue: target genes include glutathione peroxidase 3 and cell death-inducing DNA fragmentation factor, $\alpha$-subunit-like effector $A$
}

\author{
Lovisa Lundholm, Milica Putnik, Michio Otsuki ${ }^{1}$, Sandra Andersson, Claes Ohlsson ${ }^{2}$, Jan-Åke Gustafsson \\ and Karin Dahlman-Wright
}

Department of Biosciences and Nutrition at Novum, Karolinska Institutet, SE-141 57 Huddinge, Sweden

${ }^{1}$ Department of Medicine, Osaka University Graduate School of Medicine, 2-2 Yamada-Oka, Suita-City, Osaka 565-0871, Japan

${ }^{2}$ Division of Endocrinology, Department of Internal Medicine, Sahlgrenska University Hospital, SE-413 45 Göteborg, Sweden

(Correspondence should be addressed to L Lundholm; Email: lovisa.lundholm@biosci.ki.se)

\begin{abstract}
Obesity has become a major health problem in many parts of the world. Estrogens are known to reduce adipose tissue mass in both humans and animals but the molecular mechanisms are not well characterized. We used gene expression profiling to study long-term effects of estrogen on gene expression in mouse white adipose tissue and hypothalamus. Overall, the effects of estrogen on hypothalamic gene expression were much smaller than the corresponding effects on white adipose tissue gene expression. We characterize in detail estrogenic regulation of glutathione peroxidase 3 (GPX3). Our studies suggest that GPX3 is a direct estrogen receptor $\alpha$ target gene in white adipose tissue. Since obesity is correlated with
\end{abstract}

oxidative stress, and GPX3 has been demonstrated to be lower in obesity and higher after weight loss, we hypothesize that GPX3 is one important mediator of effects of estrogen in relation to fat mass. Additional genes that were affected by estrogen in adipose tissue include cell death-inducing DNA fragmentation factor, $\boldsymbol{\alpha}$-subunit-like effector A $(C I D E A)$, a gene shown to be related to body fat in mice. We conclude that estrogen has large effects on gene expression in white adipose tissue and hypothesize that GPX3 and CIDEA could be important mediators of the effects of estrogen on fat mass. Journal of Endocrinology (2008) 196, 547-557

\section{Introduction}

Obesity has become a major health problem in many parts of the world. Overweight and obesity are associated with an increased risk for diseases such as type 2 diabetes, hypertension, coronary heart disease, and certain cancers (Khaodhiar et al. 1999). The disease incidence is primarily related to a central, android fat distribution, typical for males (Blaak 2001).

Epidemiological and experimental studies link estrogen to the maintenance and distribution of body fat. This includes observations that women usually increase their body fat mass after menopause when estrogen levels decrease, and also shift to an android fat distribution (Lovejoy 2003) and that estrogen administration reduces adipose tissue mass in both humans and animals (Wade \& Gray 1979, Mattiasson et al. 2002). The molecular mechanisms for these effects are, however, not well characterized.

Estrogens exert their effects via two nuclear receptors, estrogen receptors (ER) $\alpha$ and $\beta$ (Kuiper et al. 1996, Nilsson et al. 2001). The receptors function as ligand-dependent

transcription factors that bind to estrogen-response elements (EREs) or, for example, in association with fos and jun to activator protein 1 (AP-1) sites in target gene promoters (Nilsson et al. 2001). ER $\alpha$ is the main ER mRNA expressed in mouse adipose tissue (Lundholm et al. 2004). Both ER $\alpha$ and $\beta$ mRNAs are expressed in mouse hypothalamus (Couse \& Korach 1999).

Knockout mouse models have shed light on the role of estrogen and its receptors in rodent obesity. Mice that lack aromatase (ArKO mice) are unable to synthesize endogenous estrogen and display an obese phenotype (Jones et al. 2000). A similar phenotype is observed in mice lacking ER $\alpha$ (ERKO) but not ER $\beta$ (BERKO), indicating that ER $\alpha$ is the major mediator of the effects of estrogen to reduce fat mass in mice (Heine et al. 2000, Ohlsson et al. 2000). This is further supported by the decreased body weight seen in rats after treatment with an ER $\alpha$-selective agonist propyl pyrazole triol (PPT) but not an ER $\beta$-selective agonist diarylpropionitrile (DPN; Roesch 2006).

Estrogen could influence adipose tissue mass by central and peripheral effects. Regulation via the central nervous system 
could include behavioral regulation of physical activity and feeding, where the hypothalamus has a central role in regulating appetite and satiety. There are reports on increased running wheel activity in estrogen-treated mice, primarily mediated through ER $\alpha$ (Ogawa et al. 2003). Estrogen has been shown to reduce food intake, postulated to occur partly via increased cholecystokinin signaling and negative feedback on meal size (Geary 2001). However, it has been shown that changes in food intake are not sufficient to explain the effects of estrogen on body weight and fat mass (Roy \& Wade 1977). Finally, ERKO mice develop obesity despite equal food intake as wild-type (WT) mice, demonstrating that signaling via $E R \alpha$ affects fat mass via mechanisms that are distinct from effects on food intake (Heine et al. 2000).

Studies of changes in adipose gene expression in mice in response to estrogen administration support a direct role of the adipose tissue in control of fat mass by demonstrating estrogen regulation of key mediators of lipid synthesis (Lundholm et al. 2004, D'Eon et al. 2005).

In this study, we determine estrogen-induced changes in gene expression profiles for white adipose tissue (WAT) and hypothalamus, two tissues that could be targets for the effects of estrogen on fat mass.

\section{Materials and Methods}

\section{Animals}

For the 3-week estrogen treatment study, female C57BL/6 mice were ovariectomized at 11 weeks of age. After recovery for 1 week on normal diet, the animals were kept on soy-free diet for 1 week prior to initiation of treatment and further on during the treatment. Mice were injected daily (s.c.) with $100 \mu \mathrm{g} / \mathrm{kg} 17 \beta$-estradiol $\left(E_{2}\right)$ for 3 weeks and killed at 16 weeks of age $(n=8)$. Control mice received injections of vehicle oil (olive oil, Apoteksbolaget, Göteborg, Sweden; $n=9$ ). Gonadal WAT, interscapular brown adipose tissue (BAT), and hypothalamus were collected and kept at $-80^{\circ} \mathrm{C}$. Hypothalamus was dissected as in Allan et al. (2000), retaining the entire hypothalamus including the arcuate, ventromedial, dorsomedial, and paraventricular nuclei. Hearts were collected from mice exposed to the same experimental conditions.

For the 2-, 4-, and 6-h treatment study, female C57BL/6 mice were ovariectomized at 10 weeks of age, and treated and killed at 14 weeks of age. Mice were injected (s.c.) with $100 \mu \mathrm{g} / \mathrm{kg} \mathrm{E}$ E $_{2}$ (Sigma), $5 \mathrm{mg} / \mathrm{kg}$ ER $\alpha$-selective ligand PPT; (Tocris Cookson Inc., Ellisville, MO, USA), or $5 \mathrm{mg} / \mathrm{kg}$ ER $\beta$-selective ligand DPN; (Tocris Cookson Inc.) respectively ( $n=5$ per treatment group). Control mice received injections of vehicle oil. Gonadal WAT was collected and kept at $-80{ }^{\circ} \mathrm{C}$.

Female C57BL/6 mice were sham-operated or ovariectomized $(n=5)$ at 11 weeks of age. After recovery for 1 week on normal diet, the animals were put on a soy-free diet for 1 week before they were killed at 13 weeks of age.
Gonadal WAT and interscapular BAT were collected and kept at $-80{ }^{\circ} \mathrm{C}$. Gonadal WAT was also collected and kept at $-80{ }^{\circ} \mathrm{C}$ from female $(n=4)$ and male $(n=6) \mathrm{C} 57 \mathrm{BL} / 6$ mice killed at 4 months of age, and from female knockout mice of mixed background (with no ovariectomy or treatment). ERKO mice $(n=5)$ were killed at 11-13 weeks of age and BERKO mice $(n=3)$ were killed at 13 weeks of age, together with their respective WT controls. All studies were approved by local ethical committees for animal experiments.

\section{Microarray analysis and bioinformatics}

Total RNA was prepared from gonadal WAT and hypothalamus, from individual animals, using Trizol Reagent (Invitrogen) and further purified using RNeasy Mini Kit (Qiagen). RNA was reverse transcribed into cDNA, in vitro transcribed into cRNA, and prepared for DNA microarray analysis according to the Affymetrix Gene Chip Expression Analysis manual (Affymetrix, Santa Clara, CA, USA). Affymetrix Mouse Expression Array 430A was used to profile WAT mRNA and Mouse Genome 430A 2.0 arrays to profile hypothalamus mRNA. These two types of arrays include identical probe pairs and are only distinguished by different feature formats. Three estrogen treatment samples and three vehicle samples from individual mice were analyzed for each tissue. The scanned output files were analyzed using Affymetrix Microarray Suite Version 5.0 software.

Each estrogen-treated sample was compared with each vehicle-treated sample, resulting in nine pairwise comparisons. Genes were regarded as differentially expressed when producing concordant calls (increased or decreased call) in at least seven of nine comparisons (Affymetrix Data Mining Tool Version 3.0.). In addition, genes designated as absent on all chips and genes with mixed probe sets $\left(\_x \_\right.$at, the probe sets contain at least one probe that cross-hybridizes with other sequences) were excluded from further analysis. For duplicate probe sets, only one set is reported in Table 3 to reduce the complexity of this table. In this case, probe sets are included based on the following criteria: 1. unique identifiers (_at, according to Affymetrix nomenclature), 2. highest number of present calls, 3. highest concordance, and 4. highest average fold change. In Table 3, a higher stringency was applied, only including genes regulated in all comparisons $(100 \%$ concordance). Using pairwise comparisons with a high concordance level as a filter for identifying differentially expressed genes identifies, from our experience, genes that regularly can be confirmed using an independent approach.

\section{Real-time PCR analysis}

An amount of $1 \mu \mathrm{g}$ total RNA from individual animals was reverse transcribed into cDNA using reverse transcription reagents with random hexamer primers (Applied Biosystems, Foster City, CA, USA). Analysis was performed using the ABI Prism 7700 Sequence Detection System and 7500 Real-Time PCR System (Applied Biosystems). Primer and probe 
sequences are shown in Table 1. An SYBR Green-based protocol was applied for most assays, and PCR products were further analyzed by melting curve analysis to confirm a single product. A TaqMan probe-based protocol was used for glutathione peroxidase 3 (GPX3), uncoupling protein 1 (UCP1), ER $\alpha$, and $\beta$ (ER sequences from Lundholm et al. 2004). mRNA levels were calculated using the standard curve method (user bulletin no. 2, Applied Biosystems) and normalized to $18 \mathrm{~S}$ rRNA (Applied Biosystems) or hypoxanthine ribosyltransferase (HPRT; Lengacher et al. 2004, de Kok et al. 2005).

\section{Plasmid constructs}

The mouse GPX3 promoter was amplified from mouse genomic DNA using Taq polymerase (Promega Corp). Three promoter fragments, $-3600 /-123,-3600 /-1900$, and $-2000 /-123$, were cloned into the pGL3 promoter vector (Promega Corp). The sequences of the inserted promoter fragments were verified by DNA sequencing. Expression vectors containing mouse $\mathrm{ER} \alpha$ and $\beta$ cloned into the pSG5 vector (Stratagene, La Jolla, CA, USA) were used for cotransfections.

\section{Transient transfections}

Mouse preadipocyte cells (3T3-L1) were cultured in Dulbecco's modified Eagle's medium (DMEM) containing $4.5 \mathrm{~g} / 1$ glucose (Invitrogen) supplemented with $10 \%$ calf serum, $2 \mathrm{mM}$ L-glutamine, $100 \mathrm{U} /$ penicillin per $\mathrm{ml}$, and $100 \mu \mathrm{g} /$ streptomycin per $\mathrm{ml}$. Twenty thousand cells per well were seeded in 24-well plates 16-20 h before transfection in phenol red-free DMEM (Invitrogen) supplemented with glucose corresponding to $4.5 \mathrm{~g} / 1,10 \%$ dextran-coated charcoal-treated FBS, $2 \mathrm{mM}$ L-glutamine, $100 \mathrm{U} /$ penicillin per $\mathrm{ml}$, and $100 \mu \mathrm{g}$ streptomycin/ml. An amount of $0.8 \mu \mathrm{g}$ mGPX3 promoter constructs (expressing firefly luciferase as a reporter gene) was cotransfected with $0 \cdot 2 \mu \mathrm{g} \operatorname{ER} \alpha, \operatorname{ER} \beta$, or empty pSG5 vector using Lipofectamine 2000 in OptiMem I Reduced Serum Medium according to the standard protocol (Invitrogen). A plasmid expressing Renilla luciferase under control of the thymidine kinase promoter (pRL-TK,
Promega Corp.) was used for normalization. Medium was changed after $5 \mathrm{~h}$ to charcoal-treated FBS-containing media with $10 \mathrm{nM} \mathrm{E}_{2}, 100 \mathrm{nM}$ 4-OH-tamoxifen (Tam), or vehicle (99.5\% EtOH). Cells were harvested 24 h after transfection, and firefly and Renilla luciferase activities were determined using the Dual-Luciferase Reporter Assay System (Promega Corp.) according to the manufacturer's instructions.

\section{Statistical analysis}

All values represent mean \pm s.D. When significant differences are discussed, a Student's $t$-test was used (two-tailed, twosample unequal variance).

\section{Results}

Gonadal WAT is decreased in $E_{2}$-treated mice compared with vehicle-treated mice

As previously described by Lindberg et al. (2002), mice treated with $E_{2}$ for 3 weeks displayed decreased adipose tissue mass, which was not related to a general loss of body weight. Gonadal WAT weighed $297 \pm 137$ and $156 \pm 40 \mathrm{mg}$ in the vehicle- and $E_{2}$-treated mice respectively $(P<0 \cdot 05)$. There was no difference in body weight between vehicle- and $E_{2}$-treated mice $(24 \cdot 2 \pm 2 \cdot 0$ and $25 \cdot 7 \pm 0.9 \mathrm{~g}, \quad P>0.05$ respectively).

Gene expression profiling reveals limited effects of chronic estrogen exposure on $m R N A$ levels in hypothalamus

Gene expression profiles were determined for hypothalamus of mice treated with vehicle and $\mathrm{E}_{2}$ respectively. A limited number of genes were found to be regulated in this part of the brain. At 77\% concordance (see Materials and Methods), only six genes were scored as increased and eight genes as decreased. Two and three genes were increased and decreased at $100 \%$ concordance respectively (Table 2 ). Downregulation of nuclear receptor subfamily 4, group A, member 1 (NR4A1), nerve growth factor induced clone B (NGFI-B), and Nur77) was confirmed by real-time PCR (Fig. 1).

Table 1 Primers and probes for real-time PCR

$$
\text { Forward primer }\left(5^{\prime}-3^{\prime}\right)
$$

\section{mRNA}

NR4A1

GPX3

C3

ApoCI

IRS1

UCP1

CIDEA
Reverse primer $\left(5^{\prime}-3^{\prime}\right)$

GGCTCGTTGCTGGTGTTCCATA
TCTCGCCTGGCTCCTGTTT
GCTCTCCAGCCGTAGGACATTG
TTCAACTTCTCCTTCACTTTGC
ATGAGTTAAAAGGAGGATTTGCT
CCCGAGTCGCAGAAAAGAA
TCATCTGTGCAGCATAGGACATA

Probe $\left(5^{\prime}-3^{\prime}\right)$

-
TTCTGGGCTTCCCTTCCAACCAATTTG
-
-
$\overline{-}$
ACAAACCCTTTGAAAAAGGCCGTCGG
-

Nuclear receptor subfamily 4, group A, member 1 (NR4A1), glutathione peroxidase 3 (GPX3), complement component 3 (C3), apolipoprotein CI (ApoCl), insulin receptor substrate 1 (IRS1), uncoupling protein (UCP1) and cell death-inducing DNA fragmentation factor, $\alpha$-subunit-like effector A (CIDEA). 
Table 2 Genes regulated by estrogen in hypothalamus at $77-100 \%$ concordance

\begin{tabular}{|c|c|c|}
\hline & FC & $\%$ \\
\hline \multicolumn{3}{|l|}{ Name } \\
\hline \multicolumn{3}{|l|}{ Increased genes } \\
\hline a2-macroglobulin & $2 \cdot 1$ & 100 \\
\hline Synaptotagmin-like 4 & $1 \cdot 8$ & 100 \\
\hline Prolactin receptor & $2 \cdot 2$ & 88 \\
\hline Fatty acid-binding protein 7 , brain & $1 \cdot 4$ & 88 \\
\hline Von Willebrand factor homolog & $1 \cdot 8$ & 77 \\
\hline Folate receptor 1 (adult) & $1 \cdot 7$ & 77 \\
\hline \multicolumn{3}{|l|}{ Decreased genes } \\
\hline Heat shock protein 1B & $-3 \cdot 0$ & 100 \\
\hline $\begin{array}{l}\text { Nuclear receptor subfamily } 4 \text {, group A, } \\
\text { member } 1\end{array}$ & $-1 \cdot 6$ & 100 \\
\hline FBJ osteosarcoma oncogene & $-1 \cdot 4$ & 100 \\
\hline Dual specificity phosphatase 1 & $-1 \cdot 3$ & 88 \\
\hline $\begin{array}{l}\text { Dnal (Hsp40) homolog, subfamily B, } \\
\text { member } 1\end{array}$ & $-1 \cdot 2$ & 88 \\
\hline Crystallin, $\alpha \mathrm{B}$ & $-1 \cdot 3$ & 77 \\
\hline Early growth response 1 & $-1 \cdot 3$ & 77 \\
\hline CDC-like kinase 1 & $-1 \cdot 2$ & 77 \\
\hline
\end{tabular}

$\mathrm{FC}$, fold change.

Effects of chronic estrogen exposure on WAT gene expression profiles

A large number of genes were regulated in WAT after chronic estrogen exposure; 240 genes were increased and 113 genes were decreased according to our selection criteria with a concordance level of $77 \%$ (see Materials and Methods). Seventy-nine and forty-seven genes were increased or decreased at $100 \%$ concordance respectively (displayed in

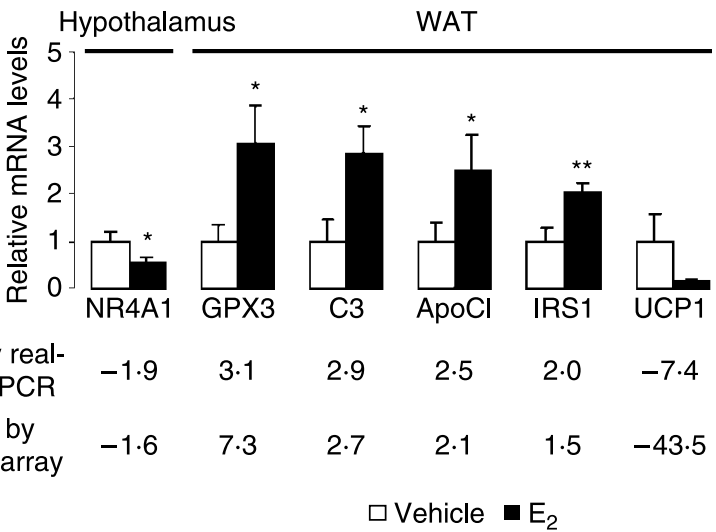

Figure 1 Confirmation of microarray data by real-time PCR, using cDNA from the same individual mice $(n=3)$ as in the microarray experiment. Real-time PCR data from hypothalamus and adipose tissue are shown in bars. Average fold changes (FC) FC from realtime PCR and microarray data respectively are presented as numbers below the bars. The hypothalamus gene was normalized to HPRT, and the WAT genes were normalized to 18S. NR4A1, nuclear receptor subfamily 4 , group $A$, member 1 ; GPX3, glutathione peroxidase 3 ; $\mathrm{C} 3$, complement component 3; ApoCl, apolipoprotein $\mathrm{Cl}$; IRS1, insulin receptor substrate 1 ; and $\mathrm{UCP1}$, uncoupling protein. ${ }^{*} P<0 \cdot 05$ and ${ }^{* *} P<0 \cdot 01$.
Table 3). (The complete list of genes changed using a filter of $77 \%$ concordance is provided as Supplementary Table 1, see Supplementary Table 1 in the online version of the Journal of Endocrinology at http://joe.endocrinology-journals.org/ content/vol196/issue3/). A subset of genes identified as regulated by estrogen in the microarray experiments were assayed using real-time PCR (Fig. 1). The estrogen regulation observed for these genes in the microarray experiment could be confirmed for all tested genes.

Additionally, several genes previously identified as being regulated by estrogen in various tissues were found to be regulated in this study. These genes included complement component C3 (Fan et al. 1996), ceruloplasmin (Middleton \& Linder 1993), and angiotensinogen (Gordon et al. 1992; Table 3).

By comparing Tables 2 and 3 , it can be concluded that the effects of chronic estrogen exposure were much greater on WAT than on hypothalamus as determined using a gene expression profiling assay. Another analysis method, significance analysis of microarrays (SAMs), also gave a clear difference in number of regulated genes between hypothalamus and WAT (data not shown). SAM assigns a score on the basis of its change in gene expression relative to the S.D. for that gene (Tusher et al. 2001). We performed this analysis using default analysis settings $(\Delta=1 \cdot 2)$. This analysis ranked 7 highly regulated genes for hypothalamus compared with 150 for WAT.

\section{Total ER $m R N A$ levels are higher in WAT than in hypothalamus}

We next investigated whether the observation that $E_{2}$ exerts greater effect on the gene expression profiles of WAT than of hypothalamus was correlated with expression levels of ER mRNAs. Studies of ER mRNA expression in these tissues revealed that $E R \alpha$ is expressed at five to ten times higher levels in WAT than in hypothalamus (Fig. 2A). ER $\beta$ is expressed at about eight times higher levels (vehicle) in hypothalamus than in WAT (Fig. 2B). However, overall, ER $\alpha$ expression is highest in both tissues. The $\mathrm{ER} \alpha / \beta$ ratio (calculated using known amounts of $\operatorname{ER} \alpha$ and $\beta$ plasmids) is $\sim 6$ in hypothalamus and several hundreds in WAT (data not shown; the ratio in WAT is in consonance with our previous report (Lundholm et al. 2004)). Importantly, the total ER mRNA levels are higher in WAT than in hypothalamus which might contribute to the quantitative differences in the effects of estrogen on gene expression profiles observed between these tissues. Figure 2 also shows that $\mathrm{E}_{2}$ treatment significantly decreased ER $\alpha$ expression in WAT and ER $\beta$ expression in hypothalamus.

\section{Genes coordinately regulated by acute and chronic estrogen} treatments in WAT

We compared the genes in WAT identified in this study as regulated by chronic administration of estrogen with genes 
that we have previously identified as regulated after $10 \mathrm{~h}$ of estrogen treatment (Lundholm et al. 2004). Thirteen genes that displayed increased expression and five genes that displayed decreased expression in this study were similarly regulated in the previous study (Table 4). Many of these genes represent novel estrogen-regulated genes.

Estrogen regulates $m R N A$ levels of GPX3, a gene involved in the response to oxidative stress

One of the genes regulated after $10 \mathrm{~h}$ and 3 weeks of estrogen exposure was GPX3 (Table 4). GPX3 was regulated at numerous time points after $\mathrm{E}_{2}$ treatment in WAT, in the liver, and in the heart (Table 5), implicating that this gene is subject to a general regulation by estrogen.

To investigate whether GPX3 is a direct estrogen target gene in vivo, mice were treated with estrogen for 2,4 , and $6 \mathrm{~h}$. As can be seen in Fig. 3A, mRNA for GPX3 was increased in vivo already after $2 \mathrm{~h}$ of estrogen treatment which is strongly indicative of direct regulation.

To determine ER specificity for GPX3 mRNA regulation in vivo, mice were treated with the $\mathrm{ER} \alpha$ - and $\beta$-selective ligands, PPT and DPN respectively for 2, 4, and $6 \mathrm{~h}$. As can be seen in Fig. 3B, GPX3 mRNA was specifically increased by the ER $\alpha$-selective ligand PPT. This regulation was significant at 4 and $6 \mathrm{~h}$, and there was a clear indication of regulation already at $2 \mathrm{~h}$ although it did not reach statistical significance $(P=0 \cdot 07)$. In addition, the levels of GPX3 mRNA in WAT were lower in ERKO mice compared with WT mice (Fig. 3C), further supporting a critical role of ER $\alpha$ in GPX3 expression in WAT. The expression of GPX3 was similar in BERKO and WT mice (Fig. 3C).

To characterize the regulation of GPX 3 transcription at the promoter level, we cloned the mouse GPX3 promoter $(-3600$ to -123$)$. This promoter fragment responded to both ER $\alpha$ and $\beta$ (Fig. 4A). However, the response to ER $\alpha$ was not ligand dependent. Division of the promoter fragment into a $-3600 /-1900$ fragment and a $-2000 /-$ 123 fragment showed that the ER response is maintained in the $-2000 /-123$ fragment. This fragment contains ERE half sites and an AP-1 site (Fig. 4B). However, mutation of these sites individually or in pair in the context of the $-2000 /-123$ fragment did not reduce the estrogen response (data not shown). Attempts to map the estrogen response within the $-2000 /-123$ fragment by deletion constructs corresponding to $-2000 /-1400,-1400 /-761$, and $-761 /-123$ respectively were unsuccessful as all fragments retained some estrogen response (data not shown). This would indicate that the GPX3 promoter is subject to a complex regulation by estrogen and in light of this it is not surprising that mutation of single or pairs of response elements did not eliminate estrogen responsiveness in the context of the $-2000 /-123$ fragment. Using less stringent criteria for prediction of EREs and AP-1 sites, sites in addition to those indicated in Fig. 4 were identified. Interestingly, 4-OH-tamoxifen activated the $\mathrm{ER} \alpha$ response in the
$-2000 /-123$ construct, while it was without effect on the ER $\beta$ response.

Cell death-inducing DNA fragmentation factor, $\alpha$-subunit-like effector $A$ (CIDEA) $m R N A$ expression is decreased by estrogen treatment in WAT

CIDEA, involved in lipid metabolism and apoptosis, was decreased by estrogen treatment (Table 3 ). We confirmed decreased expression of CIDEA by real-time PCR (Fig. 5A). A role of estrogen in the suppression of CIDEA was supported by an observed increase in CIDEA expression in estrogen-deficient OVX mice versus sham-operated mice in WAT (Fig. 5B). Further studies of CIDEA expression in WAT from animals treated for 2,4 , and $6 \mathrm{~h}$ with estrogen and ER-selective ligands did not provide evidence for CIDEA being a direct estrogen target gene (data not shown). However, the variation between different animals at these short treatment times was extensive, thus not permitting us to exclude an effect.

CIDEA and UCP1, both regarded as brown fat-specific proteins in mice, have much higher mRNA levels in female, which are the focus of this study, than in male mice WAT (Fig. 5C and D). UCP1 mRNA is also decreased after estrogen treatment in WAT (Table 3; Fig. 1).

\section{Discussion}

In this study, we show that estrogen regulates a large number of genes in WAT, which is consistent with an important effect of estrogen on this organ. In hypothalamus, on the other hand, few genes responded to estrogen treatment according to our criteria. The number of regulated genes might be related to the total ER expression in the tissues. ER $\alpha$ is the major ER expressed in both these tissues and it is expressed at higher levels in WAT than in hypothalamus. Another reason for detecting fewer changed genes in hypothalamus could be due to its more complex structure. Different regions could respond differently and changes in specific regions could be attenuated using this assay.

In WAT, there were very few genes that were equally regulated after short- and long-term estrogen treatment respectively. This is perhaps not so surprising, since time-course studies often show that genes have different temporal regulations with distinct primary, secondary, and tertiary regulation (Sismondi et al. 2007). A significant number of cytoskeletal/ motility-related mRNAs were regulated in WAT. Regulation of these could be relevant for the preadipocyte to adipocyte conversion (Takenouchi et al. 2004).

The identification of GPX3, a gene involved in the cellular response to oxidative stress, as an estrogen target gene in WAT is interesting since obesity (body mass index, BMI) is highly associated with systemic oxidative stress (Keaney et al. 2003). Furthermore, it has been demonstrated that GPX3 enzyme activity is negatively correlated to BMI; (Olusi 2002), and 
Table 3 Genes regulated by estrogen in white adipose tissue (WAT) at $100 \%$ concordance

FC

\section{Name}

Increased genes

RIKEN cDNA 9030611N15 gene

Spondin 1, (f-spondin) extracellular matrix protein Proprotein convertase subtilisin/kexin type 5

Glutathione peroxidase 3

Leucine-rich $\alpha 2$-glycoprotein

Major urinary protein 1

Angiotensinogen

Chemokine (C-C motif) ligand 8

Haptoglobin

Oxytocin receptor

Procollagen lysine, 2-oxoglutarate 5-dioxygenase 2

Growth arrest-specific 6

Complement component 4 (within $\mathrm{H}-2 \mathrm{~S}$ )

Early growth response 2

RIKEN CDNA D330035F22 gene

RIKEN cDNA $2210414 H 16$ gene

Cadherin 11

RIKEN CDNA 1300017C10 gene

Osteoglycin

RIKEN CDNA 4921521 F21 gene

RIKEN CDNA $3300001 \mathrm{H} 21$ gene

Lumican

Coagulation factor XIII, $\alpha$-subunit

Phospholipase A1 member A

Complement component 3

Neurotrophic tyrosine kinase, receptor, type 2

CD163 antigen

Gap junction membrane channel protein $\alpha$ - 1

Macrophage galactose $\mathrm{N}$-acetyl-galactosaminespecific lectin 1

UDP-Gal: $\beta$ GIcNAc $\beta$-1,3-galactosyltransferase, polypeptide 2

Contactin 1

Protease, serine, 11 (Igf binding)

RAS, dexamethasone-induced 1

Fc receptor, IgG, low affinity IIb

Complement component 1, q subcomponent, $\beta$ polypeptide

Macrophage galactose $\mathrm{N}$-acetyl galactosamine specific lectin 2

Extracellular link domain-containing 1

Expressed sequence Al132321

Mannose receptor, $\mathrm{C}$ type 1

Complement component 1, q subcomponent, $\gamma$ polypeptide

Transglutaminase 2, C polypeptide

Ficolin A

Glucose-6-phosphate dehydrogenase 2

Odd Oz/ten-m homolog 4 (Drosophila)

Insulin-like growth factor-binding protein 6

Growth hormone receptor

Folate receptor 2 (fetal)

Cathepsin C

RIKEN cDNA $4921526 G 09$ gene

Epidermal growth factor receptor

CD53 antigen

Carbonic anhydrase 5b, mitochondrial
Table 3 Continued

ATP-binding cassette, subfamily A (ABC1), member 1 SMAF1

Glycerol-3-phosphate acyltransferase, mitochondrial Amyloid $\beta$ (A4)-precursor protein

Glial cell line derived neurotrophic factor family receptor $\alpha-1$

Glucose-6-phosphate dehydrogenase X-linked $\quad 1 \cdot 8$

Phosphogluconate dehydrogenase

Glycoprotein m6b

Purinergic receptor P2X, ligand-gated ion channel 4

Acetyl coenzyme A carboxylase

RIKEN CDNA $1110032 E 23$ gene

Nuclear receptor-binding factor 1

Ceruloplasmin

Lysophospholipase 3

Melanoma antigen, family D, 1

Lysyl oxidase-like

Mus musculus, clone IMAGE:5028619, mRNA

Carboxypeptidase X 1 (M14 family)

Mus musculus, Similar to RIKEN cDNA

1700066C05 gene, clone MGC:28125

IMAGE:3980327, mRNA, complete cds

Expressed sequence Al195443

Peptidylprolyl isomerase C-associated protein

Cytochrome P450, family 4, subfamily v,

polypeptide 3

RIKEN CDNA 2810418J22 gene

$\mathrm{X}$-box-binding protein 1

Hypothetical protein LOC214597

Microtubule-associated protein 1 light chain 3

Pyruvate carboxylase

Decreased genes

Troponin I, skeletal, fast 2

Troponin C, fast skeletal

Myosin, heavy polypeptide 4, skeletal muscle

Actinin- $\alpha-3$

Myosin light chain, phosphorylatable, fast skeletal muscle

Titin

Troponin T3, skeletal, fast

Myoglobin

Actin, $\alpha-1$, skeletal muscle

ATPase, $\mathrm{Ca}++$ transporting, cardiac muscle, fast twitch 1

Myosin, heavy polypeptide 2, skeletal muscle, adult

Uncoupling protein 1, mitochondrial

Creatine kinase, muscle

Parvalbumin

Ryanodine receptor 1, skeletal muscle

Muscle glycogen phosphorylase

Ankyrin 1, erythroid

Adenylosuccinate synthetase 1 , muscle

Apolipoprotein B editing complex 2

Enolase 3, $\beta$-muscle

Tropomyosin $1, \alpha$

RIKEN CDNA $2310076 E 16$ gene

Fatty acid-binding protein 3, muscle, and heart

Titin-cap

Myozenin 1

Myosin light chain, alkali, fast skeletal muscle

Myomesin 1 
Table 3 Continued

\begin{tabular}{|c|c|}
\hline & FC \\
\hline Tropomyosin 2, $\beta$ & $-6 \cdot 6$ \\
\hline Desmin & $-5 \cdot 1$ \\
\hline Four and a half LIM domains 1 & $-5 \cdot 0$ \\
\hline Cytochrome $c$ oxidase, subunit VIIa 1 & $-4 \cdot 4$ \\
\hline Glutamate oxaloacetate transaminase 1 , soluble & $-4 \cdot 4$ \\
\hline Upregulated during skeletal muscle growth 4 & $-4 \cdot 3$ \\
\hline RIKEN cDNA $1810015 C 04$ gene & $-4 \cdot 3$ \\
\hline $\begin{array}{l}\text { Cell death-inducing DNA fragmentation factor, } \\
\alpha \text {-subunit-like effector A }\end{array}$ & $-3 \cdot 8$ \\
\hline $\begin{array}{l}\text { ATPase, } \mathrm{Ca}++ \text { transporting, cardiac muscle, slow } \\
\text { twitch } 2\end{array}$ & $-3 \cdot 8$ \\
\hline Cytochrome $c$ oxidase, subunit VIIIb & $-3 \cdot 3$ \\
\hline LIM and cysteine-rich domains 1 & $-3 \cdot 1$ \\
\hline RIKEN CDNA $1110003 B 01$ gene & $-2 \cdot 9$ \\
\hline SPARC-related modular calcium binding 2 & $-2 \cdot 5$ \\
\hline 3-Ketoacyl-CoA thiolase B & $-2 \cdot 5$ \\
\hline Lactate dehydrogenase 2 , B chain & $-2 \cdot 3$ \\
\hline Hypothetical protein MGC18894 & $-2 \cdot 1$ \\
\hline Regulator of G-protein signaling 5 & -1.9 \\
\hline $\begin{array}{l}\text { Suppressor of initiator codon mutations, related } \\
\text { sequence } 1 \text { (S. cerevisiae) }\end{array}$ & $-1 \cdot 7$ \\
\hline Cyclin D1 & $-1 \cdot 6$ \\
\hline RIKEN CDNA $4930542 G 03$ gene & $-1 \cdot 4$ \\
\hline
\end{tabular}

GPX3 mRNA was shown to be increased in obese women after a period of energy-restricted diet (Dahlman et al. 2005). GPX3 mRNA is present in many cell types (Bierl et al. 2004), but both earlier and array data indicate a high expression of GPX3 mRNA in WAT (Maeda et al. 1997; data not shown). Interestingly, two additional genes involved in the response to oxidative stress, catalase and isocitrate dehydrogenase 1 $(\mathrm{NADP}+$, soluble), were upregulated by 3 weeks of estrogen exposure (1.6- and 1.9-fold at 88 and $77 \%$ concordance respectively; Supplemental Table 1), further supporting a role of estrogen in the protection against oxidative stress. However, our additional experiments did not support that these represent direct estrogen target genes and they were not investigated further (data not shown).

We show here that GPX3 mRNA is regulated as early as $2 \mathrm{~h}$ after estrogen treatment in vivo, indicating a direct transcriptional regulation. Furthermore, GPX3 was shown to be regulated specifically via $\mathrm{ER} \alpha$ in adipose tissue, since activation was achieved using the ER $\alpha$-selective ligand PPT but not the ER $\beta$-selective ligand DPN. However, this most likely reflects the relative levels of the two ERs in this tissue rather than selective promoter regulation. We were unable to detect the GPX3 protein in WAT by western blot analysis to confirm its regulation at the protein level. However, the kidney is known to express high levels of GPX3 and in this tissue we could detect a robust increase in GPX3 following 3 weeks of estrogen exposure (data not shown).

In transient transfection assays, the GPX3 promoter was regulated by both ERs. However, ER $\alpha$ regulation of the GPX3 promoter was ligand independent in this system.
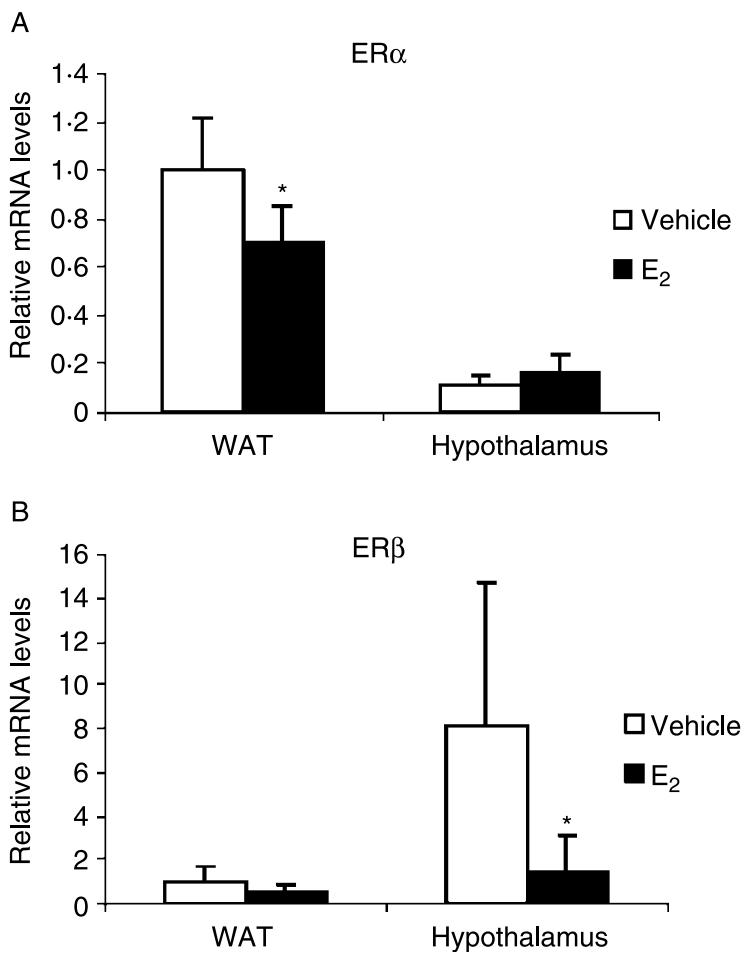

Figure 2 mRNA levels of (A) ER $\alpha$ and (B) $E R \beta$ in WAT and hypothalamus, relative to the WAT vehicle level for the respective receptor mRNA ( $n=7-9)$. mRNA was normalized to HPRT. There was a significant decrease in ER $\alpha$ mRNA in WAT, and ER $\beta$ mRNA in hypothalamus, after long-term estrogen treatment. Observe that the $E R \alpha / \beta$ ratio is several hundred fold for WAT (data not shown; see Results). $* P<0 \cdot 05$.

Decreasing the amounts of transfected ER $\alpha$ did not result in estrogen dependency (data not shown). The reason for the ligand-independent activation is unclear. Interestingly, ligand activation of $\mathrm{ER} \alpha$ was observed with 4-OH-tamoxifen (Fig. 4A). However, we have observed that both $\mathrm{E}_{2}$ and 4-OH-tamoxifen enhance activation, in the presence of transfected ER $\alpha$, of the GPX3 promoter in the mouse hepatoma Hepa cell line (data not shown). The observed activation by 4-OH-tamoxifen suggests that activation is mediated via AP-1 sites as tamoxifen has been shown to act as an agonist via such sites (Paech et al. 1997). Tamoxifen has also previously been shown to induce a stronger response than $\mathrm{E}_{2}$ in the presence of ERs on an electrophile (or antioxidant)response element (EpRE or ARE) in the human quinone reductase promoter (Montano et al. 2005). The EpRE is nearly identical to our reported AP-1-site, only differing by one base (mouse GPX3 - 1159 bp: 5'-TCTGAGTCA-3', the underlined $T$ is a $G$ in the human EpRE sequence). However, we did not observe reduced activation of the GPX3 promoter after mutation of this EpRE site (data not shown; Rushmore et al. 1991).

Regulation of GPX3 enzyme activity by estrogen has been described by L'Abbe et al. (1992). Upregulation of GPX3 mRNA in response to estrogen has been reported in uterus 
Table 4 Genes regulated after $10 \mathrm{~h}$ (experimental setup from Lundholm et al. (2004) and 3 weeks (this study) of estrogen treatment in white adipose tissue (WAT)

GO biological process description

\section{Increased genes}

Glucose-regulated protein/phospholipase C, $\alpha$

Glutathione peroxidase 3

Growth arrest specific 6

Haptoglobin

Osteoglycin/mimecan

Prostaglandin 12 (prostacyclin) synthase

Protease, serine, 11 (Igf binding)

Purine-nucleoside phosphorylase

Leucine-rich $\alpha$-2-glycoprotein/RIKEN cDNA

$1300008 B 03$ gene

Myoinositol 1-phosphate synthase A1/RIKEN

CDNA 1300017C10 gene

RIKEN CDNA 1810009A15 gene

Sec61 $\alpha-1$ subunit $(S$. cerevisiae)

Thrombospondin 1

Decreased genes

Aquaporin 1

DNA segment, human D4S114

Manic fringe homolog (Drosophila)/ $\beta-1,3-N$-acetylglucosaminyltransferase

Myocilin

Regulator of G-protein signaling 5
Previously shown $E_{2}$ regulation

LocusLink numbers were used to compare gene lists since the experiments were performed on different arrays. The column heading 'previously shown $\mathrm{E}_{2}$ regulation' refers to the fact that these genes have previously been reported to be regulated by estrogen in various cells and tissues.

and ovary after 3 days of treatment (Waters et al. 2001), and GPX3 mRNA expression was decreased in adipose tissue of ovariectomized compared with control mice (Ye et al. 2005). However, to our knowledge, this represents the first report demonstrating GPX3 as a direct target gene for estrogen. Our data are consistent with a causative effect of this gene on fat mass as GPX3 regulation occurs prior to the decrease in adipose tissue mass.

CIDEA knockout mice show a lean phenotype and are resistant to obesity (Zhou et al. 2003). The mice have markedly less WAT, as a result of reduced lipid accumulation, and increased lipolysis in BAT (Zhou et al. 2003). Similarly there is increased lipolysis in humans, in preadipocytes treated with
siRNA against CIDEA (Nordstrom et al. 2005). Previous publications have shown high expression of CIDEA in mouse BAT and expression in human s.c. WAT, but indicated that CIDEA is not expressed or expressed only at low levels in mouse WAT (Zhou et al. 2003, Nordstrom et al. 2005). However, we do observe expression in mouse WAT as determined by the Affymetrix analysis and real-time PCR. This discrepancy could be explained by a difference in CIDEA expression levels between female and male mice. Only male mice were studied in Nordstrom et al. (2005), and gender was not indicated in Zhou et al. (2003). We were unable to detect CIDEA protein in any tissue where the mRNA was regulated to confirm its regulation at the protein level.

Table 5 Average fold changes for glutathione peroxidase 3 (GPX3) mRNA assayed by microarray analysis in $17 \beta$-estradiol ( $\left.E_{2}\right)$ - versus vehicletreated mice

WAT

\section{GPX3 fold change} on microarray

$10 \mathrm{~h}$

$1 \cdot 9$
$24 \mathrm{~h}$

$1 \cdot 7$
Liver

$48 \mathrm{~h}$

$2 \cdot 3$

3 weeks
$8 \cdot 8$

3 weeks

$7 \cdot 3$
$8 \cdot 8$
Heart

3 weeks

$1 \cdot 6$

Data from different tissues of ovariectomized mice were used, and $\mathrm{E}_{2}$ treatment was performed according to the indicated time periods. The 10- to 48-h data are from the experiment described in Lundholm et al. (2004), and the liver data were previously published as supplemental data in Lindberg et al. (2003). 

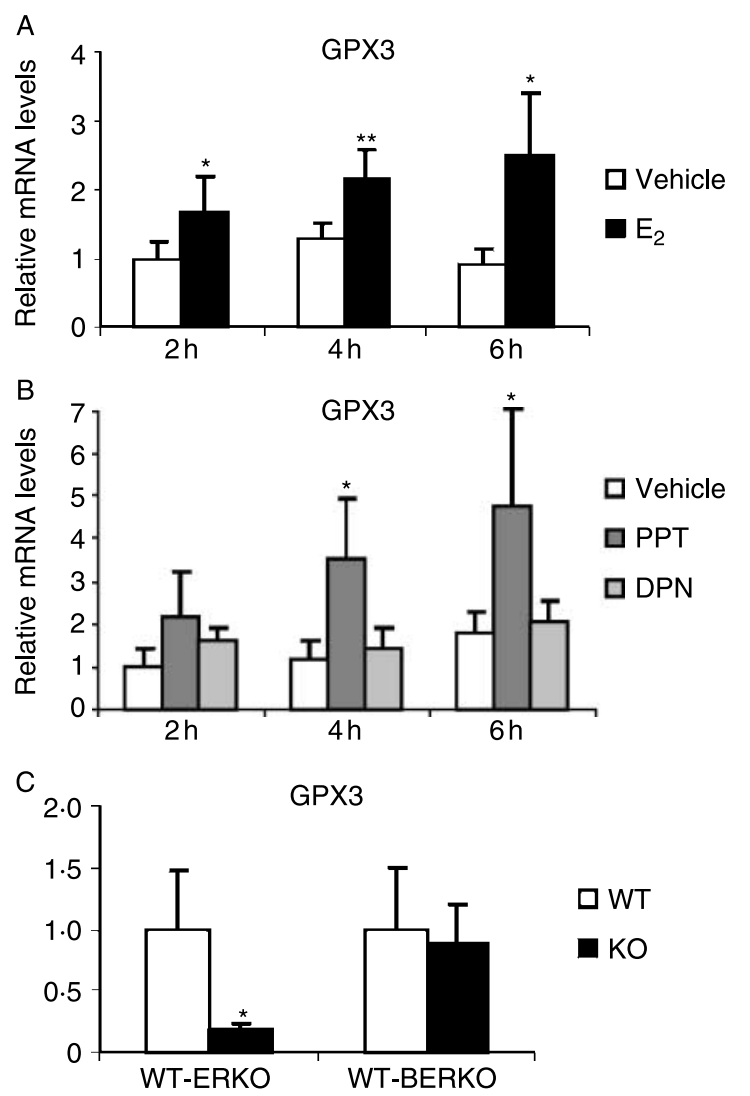

Figure $3 \mathrm{mRNA}$ expression of GPX3 is significantly increased by (A) $E_{2}$ after 2, 4, and $6 \mathrm{~h}$ and by the (B) ER $\alpha$-selective ligand PPT after 4 and $6 \mathrm{~h}$ in WAT. Mice were treated with $\mathrm{E}_{2}, \mathrm{PPT}$, or DPN and killed at the indicated time points $\left(n=5\right.$, except $6 \mathrm{~h} \mathrm{E}_{2}$ where $\left.n=4\right)$. (C) mRNA expression of GPX3 is significantly decreased in ERKO mice but not in BERKO mice compared with WT in WAT. GPX3 mRNA expression was determined using a real-time PCR assay. mRNA was normalized to HPRT. ${ }^{*} P<0 \cdot 05$ and $* * P<0 \cdot 01$.

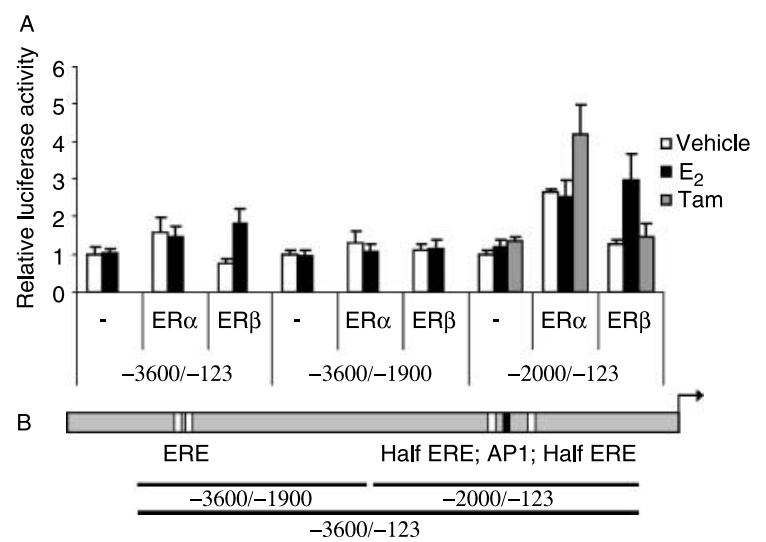

Figure 4 (A) The mouse GPX3 promoter (the $-2000 /-123$ region) is responsive to estrogen in a transient transfection assay. Cells were transfected with expression vectors ( $E R \alpha$ and $\beta$, or empty expression vectors) and reporter vectors and treated as indicated. (B) Several poss-ible ER-binding sites are present in the mouse GPX3 promoter. The marked EREs start at $-3226,-1211$, and -914 and the AP-1 site at -1159 .
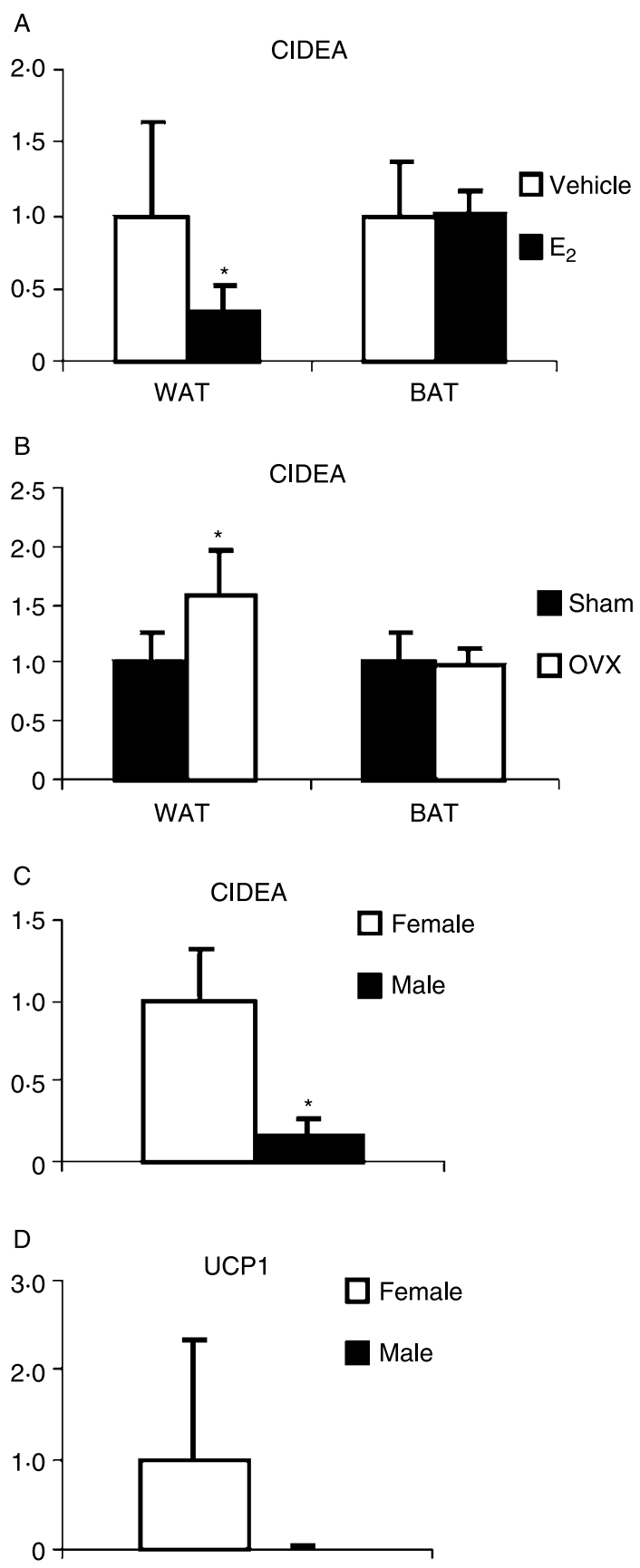

Figure 5 mRNA expression of CIDEA in WAT is significantly (A) decreased by $E_{2}$ after 3 weeks of treatment $(n=8-9)$, and similarly (B) increased in OVX mice compared with sham-operated mice $(n=5)$. mRNA expression of both (C) CIDEA and (D) UCP1 in WAT is lower in male than in female mice (UCP1 not significantly changed; $n=4-6)$. CIDEA and UCP1 mRNA expressions were determined using a real-time PCR assay. mRNA was normalized to $(\mathrm{A}$ and $\mathrm{B})$ HPRT and $\left(\mathrm{C}\right.$ and D) $18 \mathrm{~S} .{ }^{*} P<0 \cdot 05$. 
Additional genes that were identified as increased after estrogen treatment in this study include apolipoprotein CI (ApoCI) and AE-binding protein $1(2 \cdot 1-$ and $1 \cdot 6$-fold respectively at $88 \%$ concordance; Supplementary Table 1). These genes might be implicated in the reduced adiposity seen after estrogen treatment, ApoCI is involved in reducing triglyceride uptake and AE-binding protein 1 is a downregulator of adipogenesis. Mice overexpressing ApoCI have reduced adipose tissue weight, probably due to a reduction in uptake of fatty acids into adipocytes (Jong et al. 2001). When crossbred on the obese ob/ob mouse background, the transgenic mice were fully protected from development of obesity and had improved insulin sensitivity compared with normal $o b / o b$ mice (Jong et al. 2001).

Several genes involved in facilitating cholesterol and lipid efflux were increased by estrogen, including ATP-binding cassette subfamily A member 1 (ABCA1; Table 3), apolipoprotein $\mathrm{E}$, and low-density lipoprotein receptor-related protein $1(1 \cdot 7$ - and $1 \cdot 5$-fold respectively at $77 \%$ concordance; Supplementary Table 1). Interestingly, in our previous short-term study (Lundholm et al. 2004), the liver X receptor (LXR) $-\alpha$ pathway, including ABCA1 and apolipoprotein $\mathrm{E}$, was identified as decreased by $E_{2}$. The long-term effects of $E_{2}$ are apparently opposite for these two genes, but expression of LXR $\alpha$ was not changed after 3 weeks of $E_{2}$ treatment.

During these studies, the paper by D'Eon et al. was published. In our study, we do not observe decreased lipogenic genes in WAT as described in D'Eon et al. (2005). The studies differ, however, both in treatment time and mode, and access to and type of food. D'Eon et al. used pair-fed mice on standard rodent chow (not soy-free) that were treated (with $\mathrm{E}_{2}$ pellets) for about twice as long time (40 days) as in our study, and fasted before killing. In addition, Penza et al. (2006) showed that, unlike in our study, lipogenic and adipogenic genes were decreased and UCP1 was increased after $\mathrm{E}_{2}$ treatment. However, they study 4-week-old male mice compared with our studies of older female mice. Comparing their gene expression data with ours, there are about 20 genes that are similarly regulated in both studies.

Recently, ER $\alpha$ was specifically silenced in the ventromedial nucleus of the hypothalamus, with obesity as a consequence (Musatov et al. 2007). This was preceded by increased food intake but also to a decline in energy expenditure (Musatov et al. 2007). The relative role of ER $\alpha$ in central versus peripheral tissues remains to be elucidated. Still, no obvious appetite- or satiety-related genes were found to be regulated in hypothalamus in our study, although we do see a decreased adipose tissue mass.

In conclusion, we have shown that estrogen has large effects on gene expression in WAT. We have in detail characterized estrogen regulation of GPX3, a gene involved in the cellular response to oxidative stress. We hypothesize that estrogen regulation of GPX3 could be involved in protection against oxidative stress and associated phenotypes such as obesity.

\section{Acknowledgements}

This work was supported by grants from The Swedish Cancer Society, Novo Nordisk Foundation and Karo Bio AB. J-Å G is consultant and share holder of Karo Bio $\mathrm{AB}$, a campusbased biotech company working with nuclear receptor-based drug development. For the other authors, there is no conflict of interest.

\section{References}

Allan MF, Nielsen MK \& Pomp D 2000 Gene expression in hypothalamus and brown adipose tissue of mice divergently selected for heat loss. Physiological Genomics 3 149-156.

Bierl C, Voetsch B, Jin RC, Handy DE \& Loscalzo J 2004 Determinants of human plasma glutathione peroxidase (GPx-3) expression. Journal of Biological Chemistry 279 26839-26845.

Blaak E 2001 Gender differences in fat metabolism. Current Opinion in Clinical Nutrition and Metabolic Care 4 499-502.

Couse JF \& Korach KS 1999 Estrogen receptor null mice: what have we learned and where will they lead us? Endocrine Reviews 20 358-417.

Dahlman I, Linder K, Arvidsson Nordstrom E, Andersson I, Liden J, Verdich C, Sorensen TI \& Arner P 2005 Changes in adipose tissue gene expression with energy-restricted diets in obese women. American Journal of Clinical Nutrition $\mathbf{8 1} 1275-1285$.

D'Eon TM, Souza SC, Aronovitz M, Obin MS, Fried SK \& Greenberg AS 2005 Estrogen regulation of adiposity and fuel partitioning. Evidence of genomic and non-genomic regulation of lipogenic and oxidative pathways. Journal of Biological Chemistry 280 35983-35991.

Fan JD, Wagner BL \& McDonnell DP 1996 Identification of the sequences within the human complement 3 promoter required for estrogen responsiveness provides insight into the mechanism of tamoxifen mixed agonist activity. Molecular Endocrinology 10 1605-1616.

Geary N 2001 Estradiol, CCK and satiation. Peptides 22 1251-1263.

Gordon MS, Chin WW \& Shupnik MA 1992 Regulation of angiotensinogen gene expression by estrogen. Journal of Hypertension 10 361-366.

Heine PA, Taylor JA, Iwamoto GA, Lubahn DB \& Cooke PS 2000 Increased adipose tissue in male and female estrogen receptor-alpha knockout mice. PNAS 97 12729-12734.

Jones ME, Thorburn AW, Britt KL, Hewitt KN, Wreford NG, Proietto J, Oz OK, Leury BJ, Robertson KM, Yao S et al. 2000 Aromatase-deficient (ArKO) mice have a phenotype of increased adiposity. PNAS 97 12735-12740.

Jong MC, Voshol PJ, Muurling M, Dahlmans VE, Romijn JA, Pijl H \& Havekes LM 2001 Protection from obesity and insulin resistance in mice overexpressing human apolipoprotein C1. Diabetes 50 2779-2785.

Keaney JF, Jr, Larson MG, Vasan RS, Wilson PW, Lipinska I, Corey D, Massaro JM, Sutherland P, Vita JA \& Benjamin EJ 2003 Obesity and systemic oxidative stress: clinical correlates of oxidative stress in the Framingham Study. Arteriosclerosis, Thrombosis, and Vascular Biology 23 434-439.

Khaodhiar L, McCowen KC \& Blackburn GL 1999 Obesity and its comorbid conditions. Clinical Cornerstone 2 17-31.

de Kok JB, Roelofs RW, Giesendorf BA, Pennings JL, Waas ET, Feuth T, Swinkels DW \& Span PN 2005 Normalization of gene expression measurements in tumor tissues: comparison of 13 endogenous control genes. Laboratory Investigation 85 154-159.

Kuiper GG, Enmark E, Pelto-Huikko M, Nilsson S \& Gustafsson JA 1996 Cloning of a novel receptor expressed in rat prostate and ovary. PNAS 93 5925-5930.

L'Abbe MR, Collins MW, Trick KD \& Laffey PJ 1992 Glutathione peroxidase activity in a healthy Canadian population. Effects of age, smoking and drinking habits, exercise and oral contraceptive use. Trace Elements in Medicine 9 45-53.

Lengacher S, Magistretti PJ \& Pellerin L 2004 Quantitative rt-PCR analysis of uncoupling protein isoforms in mouse brain cortex: 
methodological optimization and comparison of expression with brown adipose tissue and skeletal muscle. Journal of Cerebral Blood Flow and Metabolism 24 780-788

Lindberg MK, Weihua Z, Andersson N, Moverare S, Gao H, Vidal O, Erlandsson M, Windahl S, Andersson G, Lubahn DB et al. 2002 Estrogen receptor specificity for the effects of estrogen in ovariectomized mice. Journal of Endocrinology 174 167-178.

Lindberg MK, Moverare S, Skrtic S, Gao H, Dahlman-Wright K, Gustafsson JA \& Ohlsson C 2003 Estrogen receptor (ER)- $\beta$ reduces ER $\alpha$-regulated gene transcription, supporting a 'ying yang' relationship between $\mathrm{ER} \alpha$ and ER $\beta$ in mice. Molecular Endocrinology 17 203-208.

Lovejoy JC 2003 The menopause and obesity. Primary Care 30 317-325.

Lundholm L, Moverare S, Steffensen KR, Nilsson M, Otsuki M, Ohlsson C, Gustafsson JA \& Dahlman-Wright K 2004 Gene expression profiling identifies liver $\mathrm{X}$ receptor alpha as an estrogen-regulated gene in mouse adipose tissue. Journal of Molecular Endocrinology 32 879-892.

Maeda K, Okubo K, Shimomura I, Mizuno K, Matsuzawa Y \& Matsubara K 1997 Analysis of an expression profile of genes in the human adipose tissue. Gene 190 227-235.

Mattiasson I, Rendell M, Tornquist C, Jeppsson S \& Hulthen UL 2002 Effects of estrogen replacement therapy on abdominal fat compartments as related to glucose and lipid metabolism in early postmenopausal women. Hormone and Metabolic Research 34 583-588.

Middleton RB \& Linder MC 1993 Synthesis and turnover of ceruloplasmin in rats treated with 17 beta-estradiol. Archives of Biochemistry and Biophysics 302 $362-368$.

Montano MM, Bianco NR, Deng H, Wittmann BM, Chaplin LC \& Katzenellenbogen BS 2005 Estrogen receptor regulation of quinone reductase in breast cancer: implications for estrogen-induced breast tumor growth and therapeutic uses of tamoxifen. Frontiers in Bioscience 10 1440-1461.

Musatov S, Chen W, Pfaff DW, Mobbs CV, Yang XJ, Clegg DJ, Kaplitt MG \& Ogawa S 2007 Silencing of estrogen receptor alpha in the ventromedial nucleus of hypothalamus leads to metabolic syndrome. PNAS 104 2501-2506.

Nilsson S, Makela S, Treuter E, Tujague M, Thomsen J, Andersson G, Enmark E, Pettersson K, Warner M \& Gustafsson JA 2001 Mechanisms of estrogen action. Physiological Reviews 81 1535-1565.

Nordstrom EA, Ryden M, Backlund EC, Dahlman I, Kaaman M, Blomqvist L, Cannon B, Nedergaard J \& Arner P 2005 A humanspecific role of cell death-inducing DFFA (DNA fragmentation factoralpha)-like effector A (CIDEA) in adipocyte lipolysis and obesity. Diabetes 54 1726-1734.

Ogawa S, Chan J, Gustafsson JA, Korach KS \& Pfaff DW 2003 Estrogen increases locomotor activity in mice through estrogen receptor alpha: specificity for the type of activity. Endocrinology 144 230-239.

Ohlsson C, Hellberg N, Parini P, Vidal O, Bohlooly M, Rudling M, Lindberg MK, Warner M, Angelin B \& Gustafsson JA 2000 Obesity and disturbed lipoprotein profile in estrogen receptor-alpha-deficient male mice. Biochemical and Biophysical Research Communications 278 640-645.
Olusi SO 2002 Obesity is an independent risk factor for plasma lipid peroxidation and depletion of erythrocyte cytoprotectic enzymes in humans. International Journal of Obesity and Related Metabolic Disorders 26 1159-1164.

Paech K, Webb P, Kuiper GG, Nilsson S, Gustafsson J, Kushner PJ \& Scanlan TS 1997 Differential ligand activation of estrogen receptors ER $\alpha$ and $E R \beta$ at AP1 sites. Science 277 1508-1510.

Penza M, Montani C, Romani A, Vignolini P, Pampaloni B, Tanini A, Brandi ML, Alonso-Magdalena P, Nadal A, Ottobrini L et al. 2006 Genistein affects adipose tissue deposition in a dose-dependent and gender-specific manner. Endocrinology 147 5740-5751.

Roesch DM 2006 Effects of selective estrogen receptor agonists on food intake and body weight gain in rats. Physiology and Behavior 87 39-44.

Roy EJ \& Wade GN 1977 Role of food intake in estradiol-induced body weight changes in female rats. Hormones and Behavior 8 265-274.

Rushmore TH, Morton MR \& Pickett CB 1991 The antioxidant responsive element. Activation by oxidative stress and identification of the DNA consensus sequence required for functional activity. Journal of Biological Chemistry 266 11632-11639.

Sismondi P, Biglia N, Ponzone R, Fuso L, Scafoglio C, Cicatiello L, Ravo M, Weisz A, Cimino D, Altobelli G et al. 2007 Influence of estrogens and antiestrogens on the expression of selected hormone-responsive genes. Maturitas $\mathbf{5 7}$ 50-55.

Takenouchi T, Miyashita N, Ozutsumi K, Rose MT \& Aso H 2004 Role of caveolin-1 and cytoskeletal proteins, actin and vimentin, in adipogenesis of bovine intramuscular preadipocyte cells. Cell Biology International 28 615-623.

Tusher VG, Tibshirani R \& Chu G 2001 Significance analysis of microarrays applied to the ionizing radiation response. PNAS 98 5116-5121.

Wade GN \& Gray JM 1979 Gonadal effects on food intake and adiposity: a metabolic hypothesis. Physiology and Behavior 22 583-593.

Waters KM, Safe S \& Gaido KW 2001 Differential gene expression in response to methoxychlor and estradiol through $\mathrm{ER} \alpha, \mathrm{ER} \beta$, and $\mathrm{AR}$ in reproductive tissues of female mice. Toxicological Sciences 63 47-56.

Ye P, Yoshioka M, Gan L \& St-Amand J 2005 Regulation of global gene expression by ovariectomy and estrogen in female adipose tissue. Obesity Research 13 1024-1030.

Zhou Z, Yon Toh S, Chen Z, Guo K, Ng CP, Ponniah S, Lin SC, Hong W \& Li P 2003 Cidea-deficient mice have lean phenotype and are resistant to obesity. Nature Genetics 35 49-56.

Received in final form 11 December 2007

Accepted 21 December 2007

Made available online as an Accepted Preprint 21 December 2007 